\title{
Téoros
}

Revue de recherche en tourisme

\section{Stratégies et caractéristiques des agences de voyages \\ détaillantes au début du XXI ${ }^{\mathrm{e}}$ siècle}

\section{Le cas des agences du Québec}

\section{François Bédard}

Volume 17, numéro 3, automne 1998

Pratiques contemporaines de gestion dans le domaine du tourisme

URI : https://id.erudit.org/iderudit/1072178ar

DOI : https://doi.org/10.7202/1072178ar

Aller au sommaire du numéro

Éditeur(s)

Université du Québec à Montréal

ISSN

0712-8657 (imprimé)

1923-2705 (numérique)

Découvrir la revue

Citer cet article

Bédard, F. (1998). Stratégies et caractéristiques des agences de voyages

détaillantes au début du $\mathrm{XXI}^{\mathrm{e}}$ siècle : le cas des agences du Québec. Téoros,

17(3), 44-50. https://doi.org/10.7202/1072178ar 


\section{$+\ldots . . .$. \\ Stratégies et CARACTÉRISTIQUeS DES AGENCES DE VOYAGES DÉTAILLANTES AU DÉBUT DU XX|' SIÈcle}

LE CAS DES AGENCES DU QuÉBEC

\section{François Bédard}

Les agences de voyages détaillantes - plus d'un millier au Québec avec un total d'environ 5000 emplois - voient leur rôle d'intermédiaires remis en question à la suite de l'évolution des technologies de l'information et des télécommunications. Les nouvelles technologies, principalement Internet, favorisent l'échange direct d'information entre les fournisseurs de services voyages et les consommateurs, en plus de permettre d'effectuer des transactions sans l'intermédiaire d'un agent ou d'un conseiller en voyages. Cette réalité ne peut que s'amplifier au cours des prochaines années grâce notamment à la convivialité toujours grandissante dans l'usage des nouvelles technologies. D'ailleurs, plusieurs experts considerent que l'industrie du voyage est l'un des secteurs les plus propices au commerce électronique. La menace de substitution est prise au sérieux par les leaders de l'industrie du voyage qui ont entrepris de repenser le rôle traditionnel de l'agence de voyages de manière à en assurer la pérennité.

Le présent article vise à cerner les principales stratégies que prévoient adopter les agences de voyages québécoises détaillantes au cours des prochaines années en regard des défis posés par les nouvelles technologies, ainsi qu'à définir les principales caractéristiques de l'agence de voyage du début đu XXI' siècle. L'article comprend trois parties : tout d'abord une description du contexte technologique et d'affaires dans lequel évoluent les agences de voyages, puis l'identification des principales stratégies et caractéristiques des agences de voyages d'ici 2002 et finalement, une discussion des données alimentée par des opinions d'experts.

\section{MÉTHODOLOGIE}

La définition du contexte technologique et d'affaires des agences de voyages a ćté effectuée à partir d'une revue de la documentation sur le sujet. L'identification des principales stratégies, ainsi que des caractéristiques de l'agence de voyages de demain, découle des résultats d'une enquête scientifique réalisée au cours de l'été 1998 , auprès des 1269 agences de voyages, au moyen d'un questionnaire auquel 190 agences ont répondu. Finalement, la discussion du contexte et des résultats de l'enquête est effectuée, appuyée sur les opinions d'une quinzaine d'experts de l'industrie du voyage, recueillies lors d'entrevues réalisées au printemps 1998 .

\section{CONTEXTE \\ TECHNOLOGIQUE ET D'AFFAIRES DES AGENCES DE VOYAGES}

Pour illustrer le contexte technologique et d'affaires des agences de voyages, nous rapportons les propos et les opinions contenus dans des écrits récents de cinq groupes d'intervenants qui s'intéressent à la question, soit les agences publiques ou parapubliques en tourisme, les représentants de l'industrie du voyage au Québec, les médias spécialisés en voyages, les grandes organisations internationales en tourisme et voyage et certains auteurs de la communauté scientifique en tourisme et voyage.

\section{AGENCES PUBLIQUES OU PARAPUBLIQUES EN TOURISME}

Dans son rapport intitulé Diagnostic sectoriel de l'industrie du voyage au Québec, publié en novembre 1994, le Comité sectoriel de l'industrie du voyage souligne que l'adaptation aux nouvelles technologies représente l'un des grands enjeux de l'industrie.

La menace appréhendée pour les agents de voyage du Québec se formule sous diverses questions dont en voici quelques-unes :Les consommateurs pourront-ils bientôt gever euxmêmes leurs voyages? Le rôle des conseillers en voyage a-t-il encore sa raison d'être ? Le rôle des agents tendra-t-il à se limiter à la vente ? Quels seront les véritables impacts de la technologie sur la structure de l'industrie ?

Le vice-président technologies à la Commission canadienne du tourisme (CCT), Jean B. Chrétien, écrivait en janvier 1996, dans le bulletin de la CCT, que

Le touriste a pris le contrôle en tant que consommateur au pouvoir sans précédent, fermement decide à imposer ses préférences et ses exigences à l'industrie pour le choix, l'emplacement, le prix, les dates et la fourniture des produits et services touristiques. 
Il poursuivait en soulignant que a avec ce changement, il est devenu d'autant plus nécessaire que le tourisme s'adapte à la société nouvelle où la technologie des communications n'est plus un sujet de controverse ; au contraire, elle est devenue une priorité contribuant au succès de l'industrie $\%$.

Lors des délibérations de l'atelier sur la technologie dans l'industrie touristique, tenu en septembre 1994 sous le parrainage de Tourisme Canada, l'un des conférenciers invités, M. Harry French, président d'Authentic Tour International, faisait référence à l'importance de changer l'attitude de résistance face aux technologies. Il déclarait :

Les membres de l'industrie ont l'impression que la technologie menace la façon dont ils mènent actuellement leurs opérations. Ils considèrent que leurs entreprises offrent un service personnalise et, pour eux, la technologie les écartera de ce genre de service. En fait, nous avons besoin non d'une transition sur le plan de l'attinde, mais d'une toute nouvelle attitude.

\section{REPRÉSENTANTS DE L'INDUSTRIE DU VOYAGE AU QUÉBEC}

Lors de leurs congrès annuels de 1994 et 1995, les membres de l'Association des agences de voyages du Québec (ACTAQuébec) ont échangé sur le thème des nouvelles technologies et de leurs effets sur les agences de voyages. Un des conférenciers, Philippe Le Roux, expert québécois en matière d'inforoutes, a abordé le thème de l'évolution de l'attitude des agents de voyages face aux incidences des nouvelles technologies de l'information. Il soulignait qu'il y a deux ans, la plupart des agents de voyages ne se sentaient que très peu préoccupés, voire étaient incrédules face aux conséquences que pourraient avoir les technologies de l'information sur leur chiffre d'affaires.

Il y a un an, en raison des nombreux échos médiatiques de l'avènement prochain d'Internet et des inforoutes accessibles aux consommateurs, les agents de woyages ont commencé à exprimer un sentiment de colère face à la menace de perdre des affaires à cause des technologies; on entendait dire a Les technologies ne viendront pas nous voler nos affaires $\mathrm{w}$. Ils ont adopté alors une attitude de résistance au changement. Cette année, le sentiment de colère et l'attitude de résistance semblent avoir cédé la place à la volonté de comprendre les changements et de s'organiser pour assurer l'avenir:

Dans un article paru en 1995, intitule Le futur est a nos portes, Simon Lindenbaum, responsable du Comité aviation et automatisation d'ACTA-Québec, indiquait que * depuis l'apparition du multimédia, il est possible $d^{\prime}$ avoir une agence fonctionnelle sans papier à toutes les étapes, depuis la réservation jusqu" au remisage du dossier. On parle beaucoup de la super autoroute électronique. Est-ce applicable à notre domaine ? Et comment ! [...] Personne ne songerait à utiliser en 1995, une voiture Ford modèle $\mathrm{T}$ mais on utilise encore le téléphone à cadran et la comptabilité manuelle. Ouvrez les yeux autour de vous aujourd'hui car, demain, il sera trop tard $»$.

\section{MÉDIAS SPÉCIALISÉS EN VOYAGES}

Les médias spécialisés ont largement fait écho depuis quelques années aux préoccupations exprimées par les agents de voyages en regard des nouvelles technologies. Déjà, en 1994, on trouvait dans les journaux ou les magazines des titres révélateurs de cette réalité comme le démontrent les citations suivantes :

- The Gazette, Montréal, 21 février 1994 : Be your own travel agent.

- The Globe and Mail, Toronto, 22 février 1994 : PC-based systems ease do-ityourself booking - Automated reservation services are gaining favor, especially with people who travel a beaten path.

- Bulletin Voyages, Montréal. 4 juillet 1994 : L'autoroute electronique ferat-elle disparaittre l'agent de voyages ? Lagent de voyages sera un super-technicien. Le métier évoluera mais ne disparaîtra pas.

En février 1995, André Désiront, journaliste au quotidien La Presse de Montréal, s'interrogeait à savoir si l'agent de voyages déraperait sur l'autoroute informatique ou, autrement dit, si l'autoroute de l'information ferait disparaittre l'agent de voyages. Il poursuivait en demandant, dans l'hypo- thèse où l'agent de voyages continue à exister quel sera son rôle : demeurera-til un conseiller écouté ? Ou deviendra-til un simple aiguilleur chargé de filtrer et de traiter la demande qui lui arrivera sous forme d'influx électronique ?

\section{ORGANISATIONS \\ INTERNATIONALES EN TOURISME ET VOYAGE}

Lors d'une conférence présentée en novembre 1994, M. John R. Zeemann, président-directeur général du World Travel and Tourism Council, faisait part aux membres du Cercle de tourisme du Québec de quelques réflexions relatives aux nouvelles technologies. Il déclarait que a pour maintenir leur niveau d'affaires et conserver leurs emplois, virtuellement tout le monde dans le secteur de voyage devra se familiariser avec les nouvelles technologies de l'information et d'automatisation des opérations s. Il concluait sa présentation en déclarant que \& l'environnement technologique va semer de la crainte chez certains tout en représentant de grandes occasions d'affaires pour d'autres $\%$.

Dans la revue Nouvelles de l'OMT, édition octobre 1995, M. Scott Wayne, directeur technologie à l'Organisation mondiale du Tourisme, brossait un tableau des résultats de ses recherches recentes ayant trait au tourisme et la technologie. D'entrée de jeu, il mentionnait que

ce cyberespace impalpable est quelque peu décourageant, mais il n'y a pas la de quoi se laisser intimider. En fait, pour l'industrie touristique, cela pourrait etre une formidable occasion de croissance [...] pour l'emporter sur ses concurrents, il faut être informé des tendances du marché touristique, des modeles et des approches de planification, des stratégies visant à réduire au minimum les effets négatifs et à porter au maximum les effets posinifs, des possibilites de formation dans le domaine du tourisme, de l'existence du matériel pédagogique, etc.

Une êtude, commanditée par l'Organisation mondiale du tourisme, intitulée L'impact des nouvelles technologies dans la distribution touristique et réalisée par Olivier Vialle sous la direction d'Antoine Cachin, a attiré notre attention. On y lit que 
l'automatisation de la réservation devait déboucher sur les ETDNS (Electronic Ticket Delivery Networks) ou les réseaux d'imprimantes par satellites, mais la remise en cause du caractère physique d'un billet et sa disparition à terme risquent de modifier les habitudes de distribution. Le * ticketless $*$ remet en question le processus de vente des produits aériens. [...] Les compagnies aériennes mettent en avant les gains de productivite que les agents de voyages peuvent réaliser grâce à ce nouveau mode de distribution. Conséquences pour les agences de voyages: des ventes de produits aériens plus rentables mais un risque de désintermédiation.

\section{AUTEURS DE LA COMMUNAUTÉ SCIENTIFIQUE EN TOURISME ET VOYAGE}

Simon Milne de l'Université McGill a réalisé uné étude en 1994 auprès de 40 propriétaires et directeurs d'agences de voyages. Les résultats font ressortir que $50 \%$ des personnes interviewées s'attendent a des changements importants ou imprévisibles dans leur industrie. Un quart des répondants sentent leur industrie menacée suite à des changements technologiques majeurs.

Dans un article publié dans le Journal of Vacation Marketing en 1995 et intitule The Consumer Marketing Revolution: The Impact of IT on Tourism, Marion M. Bennet affirmait qu'une révolution était en cours dans le domaine des agences de voyages à cause de l'apparition de nouveaux canaux de distribution rendus possibles grâce à des technologies telles que la télévision interactive, l'accès direct par ordinateur à des réseaux de distribution et l'implantation de kiosques multimédias.

Dans un autre article intitule Travels into the Future, paru dans le magazine Geographical en février 1995, M. Bennett déclarait : « Information technology is unlikely to eliminate traditional tourism information channels, such as the travel agent, although potentially it may erode their importance and alter their role. Rather than reducing the means by which consumers access information, it is more likely that technology will expand them, increasing convenience and broadening the dissemination of tourism information $\%$.
Philippe LeRoux et François Bédard signaient dans Téoros en 1995 un article intitulé Les agences de voyages vont-elles finir dans le fossé des inforoutes. Ils soulignaient que les agences de voyages ont une tradition d'utilisation des technologies (ex. les systèmes informatisés de réservation) qui remonte à près d'une vingtaine d'années. Elles devraient utiliser cette expérience et adopter une attitude proactive face aux nouvelles technologies, de manière à en faire un outil de développement plutôt que de les percevoir comme une menace à leur profession.

Sous la signature d'Irene Vlitos-Rowe, le Financial Times a produit en 1995 un ouvrage intitule The Impact of Technology on the Travel Industry - Developments and Trends. Cet ouvrage constitue un excellent travail de synthèse des conséquences des nouvelles technologies sur les agents de voyages. Il indique que les changements technologiques dans le secteur des agences de voyages forcent plusieurs agents à réévaluer leur rôle traditionnel et à s'orienter vers l'offre de services à valeur ajoutée ; ils seront alors rémunérés sur une base d'honoraires. Parmi les principaux enjeux pour le secteur des agences de voyages, on mentionne l'émission électronique de billets d'avion, les risques de « désintermédiation ", la réduction, voire même l'élimination progressive, des commissions pour les billets d'avion, l'urgent besoin pour les petites agences de se trouver une niche spécialisée qui leur permettra de survivre.

\section{PRINCIPALES STRATÉGIES ET CARACTÉRISTIQUES DES AGENCES DE VOYAGES D'ICI 2002}

Selon les résultats d'une enquête scientifique réalisée par questionnaire au cours de l'été 1998 auprès des agences de voyages du Québec, il est prévu que d'ici 2002, seulement la moitié $(50,8 \%)$ des agences conserveront le statu quo quant à la nature de leurs opérations actuelles. La grande majorité investiront dans l'acquisition d'outils technologiques $(87,3 \%)$ et dans la formation des conseillers en voyages $(87,8 \%)$. Les deux tiers $(67,4 \%)$ fabriqueront et vendront leurs propres produits en plus de vendre ceux des grossistes. Près des deux tiers $(63,4 \%)$ compteront davantage sur les services technologiques offerts par leur regroupement d'agences de voyages détaillantes. La cueillette d'informations sur la clientèle ira en augmentant $(79,4 \%)$ et le développement de programmes de fidélisation de la clientèle deviendra la norme $(89,4 \%)$. Environ le tiers $(31,2 \%)$ auront de nouveaux partenaires financiers. Vingt pour cent $(20,1 \%)$ ne seront plus en affaires à la suite de l'abandon ou de la vente de leur commerce (tableau 1).

Il existe une corrélation entre le chiffre d'affaires de même qu'entre le fait d'être membre d'ACTA-Québec et la stratégie relative au statu quo dans les opérations actuelles de l'agence. Plus le chiffre d'affaires est bas, plus on prévoit conserver

\begin{tabular}{|l|c|}
\hline \multicolumn{2}{|c|}{ TABLEAU 1 } \\
\hline Principales stratégies des agences de voyages d'ici 2002 & $\%$ \\
\hline Développer des programmes de fidélisation de la clientèle & 89,4 \\
\hline Investir dans la formation des conseillers en voyage & 87,8 \\
\hline Investir dans l'acquisition d'outils technologiques & 87,3 \\
\hline Augmenter la cueillette d'informations sur la clientèle & 79,4 \\
\hline Adopter un double rôlle (intermédiaire et voyagiste) & 67,4 \\
\hline Compter davantage sur les services techn. des regroupements & 63,4 \\
\hline Conserver le statu quo dans les activités & 50,8 \\
\hline Se trouver de nouveaux partenaires financiers & 31,2 \\
\hline Vendre l'agence ou cesser les opérations & 20,1 \\
\hline
\end{tabular}


le statu quo comme le démontrent les données suivantes : moins de 1 million de dollars $(64,1 \%), 1$ à 2,9 millions $(47,4 \%)$ et 3 millions et plus $(25,9 \%)$. Chez les agences membres d'ACTA-Québec, seulement $43,6 \%$ prévoient conserver le statu quo alors que chez les non-membres, $58,8 \%$ sont de cet avis.

Les agences IATA sont plus nombreuses que les non-IATA à juger probable d'éventuellement adopter les stratégies suivantes :

- Investir dans l'acquisition d'outils technologiques (IATA 91, $1 \%-$ nonIATA $80,3 \%$ );

- Investir dans la formation des conseillers en voyages (IATA $94,3 \%$ non-IATA $75,8 \%$ ) ;

- Développer des programmes de fidélisation de la clientèle (IATA 92,7\% non-IATA $83,3 \%$ ).

Les agences membres d'ACTA-Québec sont plus nombreuses que les non-membres à juger probable d'éventuellement adopter les stratégies suivantes :

- Investir dans la formation des conseillers en voyages (ACTA 94,1\% non-ACTA 81,4\%);

- Compter davantage sur les services technologiques offerts par un regroupement d'agences détaillantes (ACTA $72,3 \%$ non-ACTA $53,6 \%$ ) ;

- Augmenter la cueillette d'informations sur la clientèle (ACTA 87,3\% — nonACTA $70,9 \%$ ) ;

- Développer des progranmes de fidélisation de la clientèle (ACTA 95,1\% - non-ACTA 83,7\%).
Parmi les agences généralistes, 24,1\% des dirigeants jugent probable qu'ils vendront leur agence ou cesseront leurs opérations contre seulement $5,9 \%$ pour les agences spécialisées.

Parmi les stratégies relatives aux services à valeur ajoutée qui seront développées d'ici 2002 , cinq recueillent plus de $10 \%$ des réponses ; ce sont, par ordre d'importance, l'offre d'un programme de fidelisation $(26,2 \%)$, la spécialisation à l'intérieur d'un même créneau d'activités $(16,9 \%)$, le service à domicile et le service spécialisé (ex requo avec chacun $12,3 \%)$ et la carte de points $(10,8 \%)$, par exemple les points Air Miles (tableau 2).

Un changement significatif est prévu quant au type d'agence de voyages. Aujourd'hui, $80 \%$ des agences sont généralistes et $20 \%$ spécialisées. En 2002, on prévoit une situation à peu près inverse.

Sauf exception, l'appartenance à un regroupement chez les agences de voyages détaillantes sera généralisée, d'où la diminution importante prévue du nombre d'agences indépendantes.

Le nombre d'employés par entreprise demeurera à peu près inchangé. Comme $c^{4}$ est le cas aujourd'hui, la majorité des agences se situeront dans l'un ou l'autre des deux groupes suivants : le groupe des agences comptant de 1 à 4 employés et celui des agences en comptant de 5 à 9 . On prévoit qu'une augmentation du chiffre d'affaires n'entrainera pas une augmentation proportionnelle du nombre d'employés, et ce, grâce au gain de productivité découlant d'un usage accru des technologies.
Les firmes de télécommunication et les firmes d'informatique sont perçues comme les principaux nouveaux concurrents ou substituts des agences de voyages. Les centres d'appels et les télédiffuseurs sont également perçus comme des concurrents ou des substituts, mais dans une moindre mesure que les deux précédents.

La presque totalité des revenus des agences de voyages proviennent présentement des commissions versées par les fournisseurs. Depuis le plafonnement, en 1997, des commissions pour les vols intérieurs (Canada seulement) et transfrontaliers (Canada / États-Unis), les frais de services ou de dossiers ont commencé à faire leur apparition, surtout chez les agences spécialisées dans le marché corporatif. La majorité des répondants prévoient qu'en 2002 les commissions vont représenter moins de $50 \%$ des revenus des agences, ce qui implique une progression importante des revenus en frais de services et aussi des revenus autonomes provenant de la vente de produits exclusifs conçus par les agences elles-mêmes ou par le regroupement de détaillants auquel elles appartiennent.

Grâce à l'évolution technologique rapide dans les systèmes de réservation voyages, un forte probabilité existe que d'ici 2002 bon nombre d'entreprises, surtout celles de grande taille, et bon nombre de voyageurs individuels, particulièrement ceux à la recherche de voyages à bon marché, effectueront eux-mếmes une proportion significative de leurs réservations voyages sans recourir aux services d'un agent de voyages. Devant cette menace d'érosion d'une partie de la clientèle traditionnelle, les agences les plus dynamiques ont commencé à investir dans les nouvelles technologies et dans la formation de leur personnel, de manière à ajouter de la valeur à leurs services (amélioration du rapport qualitế-prix), tout en réduisant les coûts de fonctionnement par transaction. Ces investissements vont se maintenir au cours des prochaines années.

La presque totalité des agences de voyages souhaite que l'Office de protection des consommateurs (OPC) contingente le nombre de permis d'agences et qu'il revoie à la hausse les exigences professionnelles pour en détenir un. Bref on compte sur une réglementation pour hausser les barrières à l'entrée jugées présentement trop basses. Toutefois, comme le rôle premier de l' $\mathrm{OPC}$ consiste à protéger les 
consommateurs et non pas à régir l'industrie du voyage, il est peu probable que l'OPC acquiesce à ce souhait. Par contre, certaines barrières à l'entrée sont en train d'apparaître. Elles proviennent de différents facteurs d'ordre économique et organisationnel, dont les deux éléments les plus importants sont le plafonnement des commissions sur certains produits et l'importance des investissements à effectuer dans les nouvelles technologies.

Le plafonnement des commissions sur certains produits oblige les agences, pour demeurer rentables, à adopter un mode de fonctionnement plus efficace qu'autrefois, de manière à réduire les coûts par transaction et également à facturer des frais pour leurs services. Dans une industrie où, traditionnellement, il n'y avait pas de frais de services, il sera nécessaire de projeter une image de conseillers professionnels pour que les consommateurs acceptent de payer ces frais, d'où l'exigence pour les agences d'avoir du personnel qualifie (solide formation et expérience) avec ce que cela exige comme rémunération. En outre, il apparaît incontournable que l'agence de voyages de demain soit à la fine pointe de la technologie, ce qui représentera des investissements plus importants que par le passé.

\section{REPRÉSENTATIVITÉ DES RÉPONDANTS À L'ENQUÊTE}

Nous avons cherché à déterminer le niveau de representativité des 190 agences qui ont complété le questionnaire par rapport à l'ensemble des agences de voyages du Québec. Pour ce faire, nous avons analysé cinq variables pour lesquelles des données sur l'ensemble de l'industrie étaient disponibles, soit le chiffre d'affaires annuel moyen, la répartition du chiffre d'affaires entre les segments affaires et loisir et le niveau d'adhésion à l'ACTA, à l'IATA ou à un regroupement.

Les agences ayant participé à l'enquête ont un chiffre d'affaires de $27 \%$ supérieur à la moyenne des agences québécoises. En outre la proportion de celles-ci qui sont accréditées par IATA, membres d'ACTAQuébec et d'un regroupement d'agences détaillantes est supérieure à celle observée dans l'industrie. À la lumière de ces constats nous concluons que les 190 répondants se situent dans la moyenne supérieure par rapport à l'ensemble des 1269 agences de voyages du Québec.

Le segment du chiffre d'affaires corporatif / affaires (24 \%) est sous représenté dans l'enquête par rapport à l'ensemble de l'industrie $(37 \%)$ ). Cet écart s'explique par le fait qu'une partie importante des voyages corporatifs dans l'industrie est concentrée chez trois grands de l' industrie du voyage, soit Amex, Rider et Carlson Wagonlits.

\section{DISCUSSION}

Les entrevues réalisées auprès d'une quinzaine d'experts de l'industrie nous aident à mieux comprendre le contexte technologique et d'affaires des agences de voyages ainsi qu'à interpréter les résultats de l'enquête auprès des dirigeants d'agences de voyages du Québec.

Dans quatre ou cinq ans, la pratique du métier d'agent de voyages sera davantage exigeante. Les agents de voyages ne devront plus se définir uniquement comme des vendeurs de voyages, mais aussi comme des marchands de temps libre. Ils devront être proactifs pour aller chercher de nouveaux clients et fidéliser ceux qui leur sont déjà acquis. Les \& preneurs de commande sont appelés à évoluer, sinon ils disparaîtront. Évoluer signifie remettre en question les pratiques traditionnelles et disposer d'outils à la fine pointe de la technologie pour faire gagner du temps aux clients.

L'agence de voyages de demain sera une entreprise d'experts-conseils en voyages qui entretiendra des relations d'affaires avec ses clients, sera au fait de leurs besoins, leur prodiguera les conseils appropriés et disposera d'outils de fidélisation. Les agences qui auront du succès sont celles qui auront un plan d'affaires, un programme de formation et qui percevront les technologies comme un atout au développement et non comme une menace.

Le métier d'agent de voyages sera revalorisé. Du rôle de vendeur, l'agent reviendra au rôle de conseiller qui était le sien à l'origine. Les agents devront être mieux formés et posséder une grande culture personnelle. Les conseillers devront inspirer confiance et répondre au besoin de sécurité des clients. La notion de conseiller signifie également valeur ajoutée. Comme illustration $d^{4}$ un service à valeur ajoutée, mentionnons la touche personnalisee, les banques de données personnalisées et les * tout sous un même toit $*$.

Le secteur commercial aura toujours besoin, dans quatre à cinq ans, des agences de voyages spécialisées en affaires. L'agence de voyages loisir va également continuer d'exister. Plus sa taille sera petite, plus l'agence devra se spécialiser dans une niche, que ce soit un segment de clientèle, une destination ou un produit. Toutes seront engagées dans la course à la valeur ajoutée. Elles utiliseront des techniques multimédias pour présenter de façon virtuelle leurs produits; elles seront à l'écoute des besoins de leurs clients (le voyage est synonyme de rêve, de passion) ; elles seront en mesure de fournir rapidement des renseignements précis et des mises à jour complémentaires à celles contenues dans les brochures ou présentées sur Internet, tout cela de manière à éviter toute mauvaise surprise aux clients. Le leitmotiv sera dès lors d'offrir un service impeccable à tous points de vue pour fidéliser la clientèle.

Il y aura de petites et de grandes agences. Celles de petite taille compteront en moyenne quatre conseillers. Le personnel des grandes agences sera composé d'employés juniors et seniors ayant une spécialisation dans des thematiques comme par exemple la famille, le golf, les croisières. La sélection du personnel sera très stratégique. Les ressources humaines devront détenir une solide connaissance des nouvelles technologies. La productivité des conseillers devra augmenter. La valeur d'un conseiller en voyage résidera dans le temps qu'il fait gagner à son client et dans sa capacité à lui donner le maximum de services pour son argent.

Le professionnalisme pour un agent ou un conseiller en voyages relèvera de sa spécialisation, de sa connaissance du produit afin d'être en mesure de bien informer et de sécuriser le client (la formation des conseillers sera importante) et de sa capacité à offrir un service hors pair (fiche technique, banque de données, aide ou suivi téléphonique).

L'agent de voyages conservera un rôle dans la vente de forfaits ou de circuits spécialisés. Par contre, la distribution des forfaits simples se fera sans doute de plus en plus $\alpha$ en direct $\$$. Pour survivre, certaines agences devront done devenir des 
« concessionnair'es * d'une compagnie aérienne ou d'un voyagiste, car il ne serą pas rentable de répartir ses ventes parmi plusieurs compagnies. Les fournisseurs exigeront que le travail effectué par les agences de voyages soit de meilleure qualité.

Les regroupements d'agences de voyages détaillantes continueront à jouer un rôle prépondérant dans l'industrie. Le profil des agences sera différent d'un regroupement à l'autre, la différenciation se faisant selon les produits proposés. Les agences membres d'un regroupement jouiront d'une sécurité accrue, de bénéfices sociaux, ou de grandes possibilités d'avancement. Les regroupements continueront à se spécialiser comme agents de liaison entre les détaillants et les voyagistes. Les alliances ne comportant pas d'échange d'équité seront faibles.

Des agences vont prospérer, d'autres vont devoir fermer leurs portes parce qu'elles seront incapables de $s$ adapter au nouvel environnement technologique et d'affaires et de retenir des personnes de talent. Différentes hypothèses sont avancées. D'ici cinq ans, certains croient que $50 \%$ des agences actuelles seront regroupées sous une bannière et les autres auront fermé leurs portes. D'autres prévoient que sur un total d'environ 1000 agences présentement existantes, seules les 200 ou 300 agences les mieux structurées, offrant des produits originaux et appartenant à des chaînes nationales, vont subsister, ce qui implique la disparition des 700 ou 800 autres agences. Finalement, d'autres prédisent que $70 \%$ des agences vont réussir à s'adapter au nouvel environnement et vont continuer leurs opérations (y compris un certain nombre de petites agences indépendantes).

Les écoles de formation en tourisme et en voyages devront ajouter à leurs programmes des cours de culture générale et d'utilisation des technologies multimédias pour répondre aux besoins des agences de voyages (vente assistée par les nouvelles technologies). Les conseillers en voyages devront être bien formés en regard des technologies, car c'est grâce à elles qu'ils. seront a l'affut du savoir. Et au risque de commettre une lapalissade, il n'est pas inutile d'affirmer qu' $i$ sera essentiel que le personnel des agences de voyages en sache davantage sur le domaine du voyage que leurs clients. La formation devra éga- lement mettre beaucoup d'accent sur la fonction marketing pour que les futurs conseillers en voyages remplissent adéquatement les tâches d'un courtier qui propose aux clients des produits appropriés. répondant réellement à leurs besoins. Il y aura aussi un besoin de formation permanente. À cet égard, le rôle des regroupements ou des réseaux demeurera important.

Les technologies vont tout bouleverser d'ici quatre à cinq ans. Elles vont favoriser les transactions et non pas les compliquer, comme le craignent certains. Des agences mettront à la disposition de leurs clients qui recherchent des produits simples et qui n'ont pas besoin de conseils des SelfService Counters : des guichets automatiques à partir desquels il sera possible, à $l^{\prime}$ aide de moteurs de recherche, d'effectuer des réservations de billets ou de forfaits vers des destinations populaires, comme par exemple Paris ou la Floride (deux des destinations favorites des Québécois).

Les nouveaux venus (les compagnies technologiques telles Expedia, filiale de Microsoft), auront du succès à vendre sur Internet des billets d'avion, mais non pas des forfaits de $7000 \$$ à $8000 \$$ pour lesquels des conseils sont requis. Internet n'a pas d'avenir dans les produits à valeur ajoutée. La multiplicité et la complexité des tarifs offerts sur Internet feront en sorte que celui qui ne voyage pas souvent aura besoin d'un agent de voyages pour s'informer.

Le développement technologique viendra des joueurs majeurs de l'industrie. Les regroupements ou les chaînes vont se démarquer par l'utilisation de technologies : Internet pour le grand public, Intranet pour les membres du réseau ou de la chaîne et Extranet pour les fournisseurs et les clients importants. Le développement de systèmes spécialisés en voyages sera chose courante.

L'agent ou le conseiller en voyages devra être branché sur les nouvelles technologies pour avoir davantage de temps à consacrer à son client (converser de personne à personne). Il devra être habile à utiliser les outils d'accès à l'information (sur les pays, etc.) et à constituer des banques de données sur ses clients. Il doit en connaître plus que son client (déjà aujourd'hui, des clients effectuent des recherches eux-mêmes sur des sites Web tel Travelocity pour ne citer qu' un exemple). $L$ importance des investissements requis en technologie pour l'opération d'une agence de voyages constituera une barrière à l'entrée dans l'industrie, quoique l'on s'attende à ce que les coûts diminuent. Aux côtés de l'agence de voyages traditionnelle ayant pignon sur rue surgiront des agences virtuelles avec conseillers qui travaillent de leur domicile et sont relies à un central par Intranet ; ce type d'agence a déjà commencé à faire son apparition.

L'agent de voyages devra opter pour une spécialisation. Difficile à prendre, cette décision devra être arrêtéc en fonction de l'évolution de la clientèle et de ses goùts professionnels et personnels.

Les consommateurs ont été fascinés par les voyages à bon marchê. Ceci a amené une standardisation des voyages, mais il y a une limite a la standardisation. Les tours organisés perdront de la popularité avec la nouvelle génération. Il y aura plus de profit à faire avec la clientèle qui requiert des conseils qu'avec celle qui demande un prix.

La façon de rejoindre le consommateur changera. L'agent de voyages ne restera pas assis derrière son bureau à attendre le client ; il devra plutôt se rendre chez lui afin de le déloger de son a cocon $*$ ! Il faudra faire plus de démarchage et de contacts directs plutôt que d'attendre que le client entre dans l'agence.

Dans quatre à cinq ans, les frais de services seront courants. L'introduction du plafonnement des commissions, tout d'abord par les compagnies aériennes puis par les voyagistes, aura préparé le terrain à ce changement. Chez certaines agences, l'introduction des frais de services s'effectuera de façon progressive en prenant en compte le contexte concurrentiel. Les agents se woudront des professionnels et trouveront normal de demander des frais ou des honoraires pour fournir de l'information et effectuer un travail de qualité. Les frais de services et l'utilisation des nouvelles technologies contribueront à créer une image professionnelle. Des frais ou des honoraires seront facturés dans différentes situations telles que l'émission de billets d'avion de moins de $250 \$$, les réservations d'hôtel sans billet d'avion, les réservations non informatisées d'hôtel ou de voiture, la demande d'un passeport ou d'un visa 
et la préparation d'un itinéraire. La clientèle corporative sera plus réceptive à cette nouvelle formule que la clientèle loisir.

La taille d'une agence, d'un réseau ou d'un regroupement est importante pour réaliser des économies d'échelle et pour obtenir crédibilité et respect dans toute l'industrie. Il est important d'appartenir à une chaîne pour jouir de commissions bonifiées. Si les commissions diminuent gráduellement, il faudra trouver des solutions alternatives comme, par exemple, dévèlopper des forfaits de groupe avec profit ou vendre des produits et des accessoires complémentaires aux voyages. L'industrie pourrait revenir à ce qui se faisait il y a 12 à 15 ans, c'est-à-dire offrir à la clientèle des forfaits tout compris avec $1^{\dagger}$ aide des consolidateurs.

\section{CONCLUSION}

Les nouvelles technologies ont déjà commencé et vont continuer au cours des prochaines années à bouleverser le rôle traditionnel d'intermédiaires joué par les agences de voyages. Quoique les spécialistes prévoient une diminution du nombre d'agences, particulièrement chez les petites agences indépendantes, aucun ne prévoit leur disparition pure et simple en raison d'une substitution au profit de la distribution en direct, qui est facilitée par l'évolution rapide du commerce électronique, ou au profit de nouveaux intervenants dans l'industrie du voyage comme les entreprises d'informatique et de télécommunications.

Les leaders dans l'industrie des agences de voyages - principalement les regroupements d'agences détaillantes et les grands groupes intégrés — ont déjà pris des mesures pour s'ajuster aux menaces et aux défis posés par les nouvelles technologies. La stratégic consiste essentiellement à utiliser au maximum ces mêmes technologies pour augmenter la productivité et la rentabilité, à développer des services à valeur ajoutée et des outils de fidélisation et, finalement, à créer des produits exclusifs qu'ils vendront à leur clientèle en sus de ceux des voyagistes. Bref, la stratégie vise à ce que les agences de voyages soient plus que de simples intermédiaires en renforçant, d'une part, leur rôle de conseillers-experts et en devenant, elles aussi, dans une certaine mesure, des fournisseurs de services.

François Bédard est professeur au Département d'etudes urbaines et touristiques et associe de recherche à la Chaire de tourisme, Ecole des sciences de la gestion, Universite du Québec à Montráal. Son enseignement et ses activites de recherches portent entre autres sur la distribution et les nouvelles technologies en tourisme. Titalaire d'un MBA, il est à compléter la rédaction d'une thèse de doctorat à l'Université Paris I Pantheon-Sorbonne ayant comne titre : Modeles d'adaptation des agences de voyages québécoises aux nouvelles technologies pour assurer leur survie à long terme \%.

\section{RÉFÉRENCES}

Archambault, Michel, et Paul Arsenault (1994), Diagnostic sectoriel de l'industrie du voyage au Québec, Rapport final présenté par le Comité sectoriel de l'industrie du voyage, Montréal.

Bédard, François et Philippe Le Roux (1995), $\alpha$ Les agences de voyages vont-elles finir dans le fosse des inforoutes $\$$, Teoros, vol. 14, $\mathrm{n}^{*} 3$.

Bennett, Marion M. (1995), * The Consumer Marketing Revolution ; The Impact of IT on Tourism *, Journal of Vacation Markering, vol. $1, n^{\circ} 4$, p. $376-382$.

Bennett, Marion M. (1995), * Travels into Future », Geographical, p. 18-19.

Chrếtien, Jean B. (1996), La CCT vient en aide au tourisme grâce à la technologie, Communiqué, Commission canadienne du tourisme, p. 6.

Désiront, André (1995), * L'agent de voyages dérapera-t-il sur l'autoroute informatique ? *, La Presse, 18 février, Montréal.

Le Roux, Philippe (1996), Tourisme : un aller simple pour Intermet. Conférence présentếe le 28 mars, Montréal.

Lindenbaum, Simon (1994), \& Lagent de voyages sera un super-technicien! Bu Bulletin Voyages, 30 avril, p. 9.

Vialle, Olivier et Antoine Cachin (1995). L'impact des nouvelles technologies dans la distribution touristique, Organisation mondiale du tourisme, Madrid.

Vlitos-Rowe, Irene (1995), The Impact of Technology on the Travel Industry. Developments and Trends. London, Pcarson Professional Ltd (Financial Times Management Reports).

Wayne, Scott (1995), * Tourisme et technologie : le monde à portée de clavier $\%$. Nouvelles de I'OMT, octobre, $\mathrm{n}^{5}$ 5, p. 16-17.

ZEEMAN, John R. (1994), The Impact of New Technologies on Travel and Tourism Distribution. An Address to Le Cercle de tourisme du Québec, Montréal, 17 novernbre. 